\title{
The Development of Anticipation: A Cross-Sectional Examination of the Practice Experiences Contributing to Skill in Cricket Batting
}

\author{
Juanita Weissensteiner, ${ }^{1-3}$ Bruce Abernethy, ${ }^{1,4}$ \\ Damian Farrow, ${ }^{3}$ and Sean Müller ${ }^{5}$ \\ 1 University of Queensland; ${ }^{2}$ Cricket Australia; \\ ${ }^{3}$ Australian Institute of Sport; ${ }^{4}$ University of Hong Kong; \\ ${ }^{5}$ Royal Melbourne Institute of Technology
}

\begin{abstract}
The link between the anticipation skills of cricket batsmen and their practice histories was examined. Skilled and lesser skilled batsmen of U15, U20, and adult age completed a temporal occlusion task, in which they were required to use prerelease kinematic information to predict the type and length of delivery being bowled, and a structured interview, in which their accumulated hours of experience in organized and unorganized sporting activities were estimated. Skilled adult and U20 players showed an ability to use prerelease kinematic information to anticipate ball type that was not evident among any other group, and skilled players of all ages were distinguishable in terms of their accumulated hours of cricket-specific experience. Hours of cricket-specific practice, however, explained only a modest percentage of the variance in anticipatory skill. Discussion focuses upon future refinements to the measurement of anticipation and practice history plus the role that variables other than the quantum of cricket experience may play in developing anticipation.
\end{abstract}

Keywords: expertise, development, visual-perceptual skill

Even though anticipation has been well established for many years as one of the hallmarks of expert performance in time-constrained activities (e.g., Bartlett, 1947), comparatively little is still known about how expert anticipation is developed. In this article, we explore this issue within the fast ball sport of cricket. We measure the differences in anticipatory capability between expert and nonexpert

\footnotetext{
Weissensteiner is with the School of Human Movement Studies, University of Queensland; Cricket Australia; and Australian Institute of Sport; Abernethy is with the Institute of Human Performance, University of Hong Kong, and the School of Human Movement Studies, University of Queensland; Farrow is with Sports Science and Sports Medicine, Australian Institute of Sport; and Müller is with the Discipline of Exercise Sciences, Royal Melbourne Institute of Technology.
} 
cricket batsmen of different ages using an established laboratory method (Müller, Abernethy, \& Farrow, 2006) and then seek to determine the extent to which these differences in anticipatory skill are attributable to differences in the participant's developmental and practice experiences.

A number of existing studies from cricket have demonstrated that more skillful adult batsmen are able to make better predictions of ball direction and delivery type from the prerelease movement patterns of both slow (spin) and fast (pace) bowlers (e.g., Abernethy \& Russell, 1984; Müller et al., 2006; Renshaw \& Fairweather, 2000). For example, Müller et al. (2006) investigated the anticipatory skill of adult-aged expert, intermediate, and novice batsmen using a video-based occlusion paradigm involving deliveries from two accomplished bowlers. The expert batsmen were found to be superior in anticipating ball type before ball release, indicating that they could effectively extract early information, particularly from the motion of the bowling hand and arm. When facing the faster bowler, the experts relied on information available between the time of the bowler's front foot impact and their release of the ball for anticipation. For the slower (spin) bowler, the experts were able to use prerelease information available from between the time of the bowler's back foot and front foot impact for successful prediction, whereas the less skilled players were reliant exclusively upon the later ball flight information.

It has been suggested that experts may use anticipation to circumvent the reaction time delays inherent in the batting task (McLeod, 1987) and optimize their visual search strategies to make best use of early information (Land \& McLeod, 2000). Skilled batsmen appear to use information from the bowler's prerelease movement pattern to commence aspects of their response earlier and thus create the impression of "having all the time in the world" (Bartlett, 1947, p. 836) in which to execute their hitting response. The ability to identify and use advance information arising from the bowler's prerelease movement pattern may afford the batsman more time to prepare, initiate, and execute an appropriate shot, and this may be of particular importance when facing bowlers at an elite level. The expert advantage in anticipatory performance in video tasks is paralleled by the experts' superior judgments of ball length and bat-ball interception when performing actual batting under conditions of unpredictable visual occlusion (Müller \& Abernethy, 2006). What is unclear, however, is how and when the experts' anticipatory skill is developed and what developmental and practice experiences contribute to this development.

There is currently only very limited research examining the development of perceptual skills, such as anticipation, in fast ball sports such as cricket. The studies that used age as an independent variable suggest that the perceptual skills that are known to be critical for expert performance in adults improve not with maturation or chronological age alone but with experience with, and exposure to, vast amounts of task-relevant practice. Abernethy (1988) employed a film-based occlusion paradigm to investigate the effect of skill and chronological age on anticipatory performance in badminton. Groups of experts and novices of 12, 15, 18 years and adult age were used. The experts, unlike the novices, showed a progressive increase in the use of early movement pattern information as they got older, although it was only at the adult level that the anticipatory performance of the experts was significantly superior to that of the age-matched controls. Tenenbaum, 
Sar-El, and Bar-Eli (2000), in a comparable study of anticipatory skill in tennis, reported that while expert/novice differences in anticipation were evident from as early as 8-11 years of age, the magnitude of skill-related differences was greatest after some 6-7 years of accrued experience. Ward and Williams (2003) examined the relative contribution of visual, perceptual, and cognitive skill to the development of expertise in soccer. Groups of elite and subelite soccer players ranging in age from 9 to 17 years were presented with a variety of tasks including pattern recall, anticipation, and an examination of their use of situational probability information. Results suggested the emergence of skill and/or age differences may be dependent upon the type of task used to measure anticipation.

One means of gaining some insight into how perceptual-motor expertise is acquired, and to help ascertain what conditions may be essential to promote the development of expertise, is to examine the practice histories of players of different skill levels using tools such as interviews, training diaries, and questionnaires (Starkes \& Ericsson, 2003; Williams \& Ericsson, 2005). Initial work using this approach has focused on quantifying the amount of deliberate practice required to become an expert and has been motivated by observations from other domains, such as playing chess and musical instruments, that the amount of deliberate practice is monotonically related to task performance (Ericsson, Krampe, \& TeschRomer, 1993; Ericsson, 2003). This finding has also been substantiated in some sport settings (e.g., Helsen, Starkes, \& Hodges, 1998; Helsen, Hodges, Van Winckel, \& Starkes, 2000; Hodges \& Starkes, 1996). Other work investigating the personal and sociodevelopmental backgrounds of performers has indicated that sporting experts not only partake in large amounts of deliberate practice but also engage, from an early age, in vast amounts of what Côté et al. have termed deliberate play (Côté, 1999; Côté, Baker, \& Abernethy, 2007; Côté \& Hay, 2002). Deliberate play involves activities (such as informal neighborhood games, like backyard cricket) that have as their principal driver enjoyment and immediate gratification rather than performance improvement and long-term skill acquisition. Like deliberate practice, hours spent in deliberate play during development may be critical in determining whether expertise ultimately emerges. Time invested in backyard cricket, for instance, may be advantageous to the long-term development of batting expertise because, with the distance from bowler to batsman frequently being closer than normal, the task constraints may be such as to promote the early development and use of anticipatory strategies.

There are likely multiple pathways possible to expert performance (Côté et al., 2007). Côté et al.'s developmental model of sports participation (Côté, 1999; Côté \& Hay, 2002) proposes a pathway toward elite performance that consists of three distinct stages: the sampling years (childhood; age 6-12), the specializing years (early adolescence; age 13-15), and the investment years (late adolescence; age 16+). During the sampling years, athletes participate in a variety of sports with the focus being primarily on deliberate play activities. The specializing years involve engaging in fewer sporting activities-both organized and unorganizedthat are more competitive. Finally, the investment years demonstrate a commitment to only one predominantly organized sport activity. There is some evidence from team sports, at least, to suggest that a broad experience of a range of different sports during the sampling years may be advantageous. Baker, Côté, and Abernethy (2003), for example, observed a negative correlation between the number of 
sports experienced during the sampling years and the hours of practice needed in the specializing and investment years to attain national open-age selection.

The laboratory-based research on anticipation and its age-related development and the work on the practice and developmental histories of experts have largely occurred in parallel with little attempt to converge the two lines of inquiry. Detailed practice histories are typically not available to help explain individual differences in anticipatory skills tests, especially for performers of different age and skill, and, when practice histories are collected, these are typically done with respect to the global development of expertise rather than the more specific development of component skills like anticipation. The purpose of this article is to provide some convergence of the experimental and practice history approaches by exploring the putative links between the developmental and practice histories of cricket batsmen and their proficiency in sport-specific anticipation.

We employ a cross-sectional research design involving skilled and less skilled batsmen of three age groups (under 15 , under 20, and adult). We determine, in turn, the participant's anticipatory skill (using the temporal occlusion task of Müller et al., 2006) and their practice and developmental histories (using a structured interview and retrospective recall). We then examine the extent to which anticipatory skill is predictable from practice/developmental variables. We hypothesized, in relation to anticipatory skill, that the skilled batsmen would be capable of earlier and more accurate prediction of delivery type and length and this would be evident, following Müller et al. (2006), in terms of a selective ability to pick up useful predictive information in the time period from front foot impact to release. Furthermore, in line with Abernethy (1988), we hypothesized that the expert advantage would be established by adult age but perhaps no earlier. This tentative prediction was based on two considerations in relation to the likely relative importance of anticipation compared with biological/anthropometric factors in determining success at different ages, namely, (1) that the bowling speeds faced by batsmen in the junior ranks may not necessarily require anticipation for success and (2) that factors other than anticipation, such as relative age and strength and maturity levels, may be particularly important in determining junior success in cricket, as they are in many other sports (see Musch \& Grondin, 2001 for a review).

In relation to practice histories, it was expected that skilled batsmen would invest more time than lesser skilled batsmen in organized cricket-specific activities as well as in other sports in which transfer of anticipatory skill to cricket batting skill may be possible (i.e., other fast ball sports in which early "reading" of the opponent's intention is needed for successful responding). Based on Côté et al.'s (2007) developmental model of sport participation, it was predicted that these differences would emerge in the specializing years at approximately 13 to 15 years of age. When differences in age were accounted for, greater hours of involvement in cricket and cricket-related activities, organized or unorganized, were expected to be associated with superior anticipatory skill and to account for substantial interindividual variance in anticipatory skill. 


\section{Method}

\section{Participants}

A total of 102 cricket batsmen, from six different skill and age groupings, participated. All participants were male. Skilled and lesser skilled participants were formed in three age divisions - under 15 years (U15), under 20 years (U20), and adult ( $>20$ years). The skilled batsmen were those who had attained state and/or national representation. The age range of the skilled U15 batsmen was 11.4-15.0 years $(n=21, M=13.51, S D=1.20)$; the skilled $\mathrm{U} 20$ batsmen, $15.5-19.6$ years $(n$ $=18, M=17.40, S D=1.50)$; and the skilled adult batsmen, $20.3-26.5$ years $(n=$ $13, M=22.69, S D=2.19)$. The lesser skilled batsmen were those who had not attained regional representation but nevertheless had years of playing experience comparable to that of those in the age-matched skilled group. The age range of the lesser skilled U15 batsmen was $10.3-14.9$ years $(n=20, M=13.44, S D=1.21)$; the lesser skilled U20 batsmen, $15.2-19.7$ years $(n=20, M=17.74, S D=1.47)$; and the lesser skilled adult batsmen, 20.7-37.8 years $(n=10, M=26.91, S D=$ 5.76). The age groupings were chosen such that the U15 age group covered the typical sampling and specializing years, the U20 age group covered the typical investment years, and the (skilled) adult group provided the benchmark for mature performance on the task. Institutional approval of the protocol was granted and informed consent from all participants was obtained before the commencement of testing.

\section{Procedures}

Participants undertook two discrete sets of tasks - a laboratory-based task to measure anticipatory skill in cricket and a structured interview in which measurement was made of the participant's practice and developmental histories. Completion of the two sets of tasks took, on average, some $1 \mathrm{hr} 40 \mathrm{~min}$ to complete-approximately $20 \mathrm{~min}$ for the anticipation task and $80 \mathrm{~min}$ for the structured interview.

Measurement of Anticipatory Skill. The temporal occlusion task developed by Müller et al. (2006; Experiment 1) was used to assess the anticipatory skill of the participants, although only the trials displaying the swing bowler were presented. The task involved the presentation of a total of 48 trials in which the run-up and delivery action of a swing bowler was visible from the perspective of the batsman. The bowler, who was of first-class playing standard, had earlier been filmed from the batsman's perspective while bowling a series of different deliveries using his normal action. Presentation of vision of the bowler's run-up and delivery action was via a $105-\mathrm{cm}$ plasma screen. Each trial was randomly occluded at one of four possible points: at the time of the bowler's back foot impact (BFI), at the time of the bowler's front foot impact (FFI), at the time of ball release (R), or at a time after the ball had bounced and no further flight of the ball was visible (NO). The participants, on each trial, were given the three-choice task of judging whether the 
delivery they were viewing was a full outswinger, a full inswinger, or a short ball. All three delivery types were presented with equal frequency and in the same random order for all participants, and responses were made by ticking the appropriate cell on a response sheet within the 5-s intertrial interval. Participants were initially shown three unoccluded trials of each ball type, followed by two trials in which the display was occluded before ball flight being visible, with feedback on the correct responses provided after each of the practice trials. The trials shown were not ones that were included within the test and during the test no feedback was provided.

Measurement of Practice and Developmental Histories. A structured interview was conducted with each participant and the retrospective recall method (Côté, Ericsson, \& Law, 2005) was employed to generate estimates regarding the breadth and extent of their participation in organized and unorganized sporting activities. Through prompts provided by the use of developmental charts of the type used by Baker et al. (2003), participants were required to list all organized and unorganized sports activities they had participated in for each year from the age of five through to the present. Participants were asked to estimate how many hours in a typical week they would spend in each of the listed activities. To minimize positive bias and inaccurate memory recall, participants were instructed to start by completing the charts relating to their current age and then work backward (Côté et al., 2005). Organized sports activities were formal competitions, team and small group training sessions, and all 1-on-1 sessions with a coach. Unorganized activities were those where there was no formal competition or training and no involvement of a coach and consisted of activities such as solo bat and ball drills, participation in neighborhood games ("backyard cricket"), informal matches and "practices" with friends at a local park, and beach cricket. The distinction drawn between organized and unorganized activities is different from that between deliberate practice and deliberate play. For example, experience in formal competition, which is thought to be important in the development of cricket skill, does not count as either deliberate practice or deliberate play, but is captured by an organized-unorganized activity distinction. For this reason, this study was designed to examine organized vs. unorganized activities rather than the deliberate practice-deliberate play distinction.

To assess reliability, repeat interviews were conducted with two of the skilled participants and two of the less skilled participants some 30-36 months after the initial interviews. Percentage agreements between the number of organized sports, total hours of involvement in organized cricket, and total hours of involvement in unorganized cricket reported on the two occasions were $87.5 \%, 95.9 \%$, and $89.8 \%$ respectively. The comparable values for unorganized activities were 93.8\%, $85.9 \%$, and $88.6 \%$ respectively. To determine concurrent validity, the estimates of critical practice measures were compared between the reports given by the four participants and independent reports given by one of each of the participant's parents. The percentage agreements between the participants' and parents' estimates of number of activities, total hours of cricket and total hours of noncricket were $100.0 \%, 97.0 \%$, and $98.0 \%$ respectively for organized activities and $87.5 \%$, $93.1 \%$, and $95.3 \%$ respectively for unorganized activities. 


\section{Analysis of Data}

Anticipatory Skill. Following the procedures adopted by Müller et al. (2006), two dependent measures were calculated for each participant for each occlusion condition - the percentage accuracy in predicting ball type and the percentage accuracy in predicting ball length. The former was based on the accuracy in discriminating the outswingers from inswingers for the 32 full-length deliveries that were presented, while the latter was based on discriminating the "short-length" deliveries from the "full-length" deliveries across all 48 trials. Following an arcsine transformation, both percentage accuracy measures were subjected to two sets of analyses specifically designed to examine the hypothesized differences in advance information pickup between the groups of different ages and skill levels. These analyses focused on the two occlusion conditions (R and FFI) that had revealed differences in anticipatory performance in Müller et al.'s (2006) study between highly skilled and less skilled adult players. No statistical analyses were conducted on the data collected in the BFI and NO occlusion conditions.

The first analyses used a $2 \times 3$ (skill $\times$ age) factorial analyses of variance to compare the prediction accuracy of each of the groups under the condition (R) where occlusion occurred at the time of ball release. This occlusion condition was selected for the comparison of the groups because it provided the best single point to measure overall anticipatory performance (in that it was the only occlusion point at which all advance but no ball flight information was available) and significant differences in prediction of both ball type and length between highly skilled and less skilled adult players have been previously shown at this occlusion point (Müller et al., 2006). Partial eta-squared $\left(\eta_{\mathrm{p}}{ }^{2}\right)$ values were computed to determine the proportion of total variability attributable to each factor or combination of factors. Following usual convention, $\eta_{\mathrm{p}}{ }^{2}$ values $<.04$ were considered small; those in the range .05-.14, medium; and those >.14, large (Cohen, 1988). The sources of any significant effects within the ANOVA models were sought using the Tukey HSD procedure.

The second analysis examined information pickup across the time window, from front-foot impact to release (FFI-R), in which Müller et al. (2006) had previously demonstrated a selective and unique advantage for highly skilled adult batsmen. We assessed the evidence for information pickup across the FFI-R time window for each group by using planned comparisons of the prediction accuracies at FFI and R in combination with comparison of the attained prediction accuracy levels against the levels that could be attained simply through guessing. Two conditions needed to be satisfied for a conclusion that information pickup had occurred from FFI-R: (1) a significant reduction in prediction error was needed from FFI to $\mathrm{R}$ (as assessed by a one-tailed $t$ test) and (2) the prediction error at $\mathrm{R}$ needed to be superior to guessing levels. In the case of type judgments, this required prediction accuracy significantly greater than $50 \%$, whereas for length judgments this required predication accuracy significantly greater than $66.7 \%$. (For length, simply guessing "full" to all deliveries would have resulted in a correct response on two out of three trials.) The statistical comparisons against chance were made through the calculation of the $95 \%$ confidence limits for each group's accuracy score at each occlusion condition. 
Practice and Developmental Histories. Estimates of hours spent participating in different types of sporting activities were collated from the yearly practice history charts completed by each participant. The cumulated hours of practice were determined at three different levels, as illustrated in Figure 1. Total hours were calculated for organized and unorganized activities and, within each of these major distinctions, for cricket and noncricket activities. Hours spent in noncricket activities were in turn subdivided into four categories based on Schmidt and Wrisberg's (2000) transferable elements system and the extent to which the different activities may share movement/biomechanical, perceptual and/or conceptual/strategic elements in common with those required in cricket batting. Separate categorizations were made for open upper limb interceptive sports (sports such as softball, baseball, tennis, squash, volleyball, and badminton that require interception of a moving ball with a bat, racquet, or stick manipulated by the player's upper limbs); closed upper limb interceptive sports (sports such as golf and tee ball that require interception of a stationary ball using a bat, racquet, or stick manipulated by the player's upper limbs); team invasion ball sports (sports such as basketball, football, netball, hockey, soccer, handball, and water polo that have as their object scoring goals, crossing lines, or penetrating into the opposition's territory); and other sports (such as athletics, swimming, surfing, body surfing, wakeboarding, motor sport, cross country running, karate, tae kwon do, jujitsu, cycling, boxing, gymnastics and archery that do not require upper or lower body interception or team-based invasion).

The total cumulative hours spent in different types of activities plus the total number of different sporting activities experienced by each group of participants

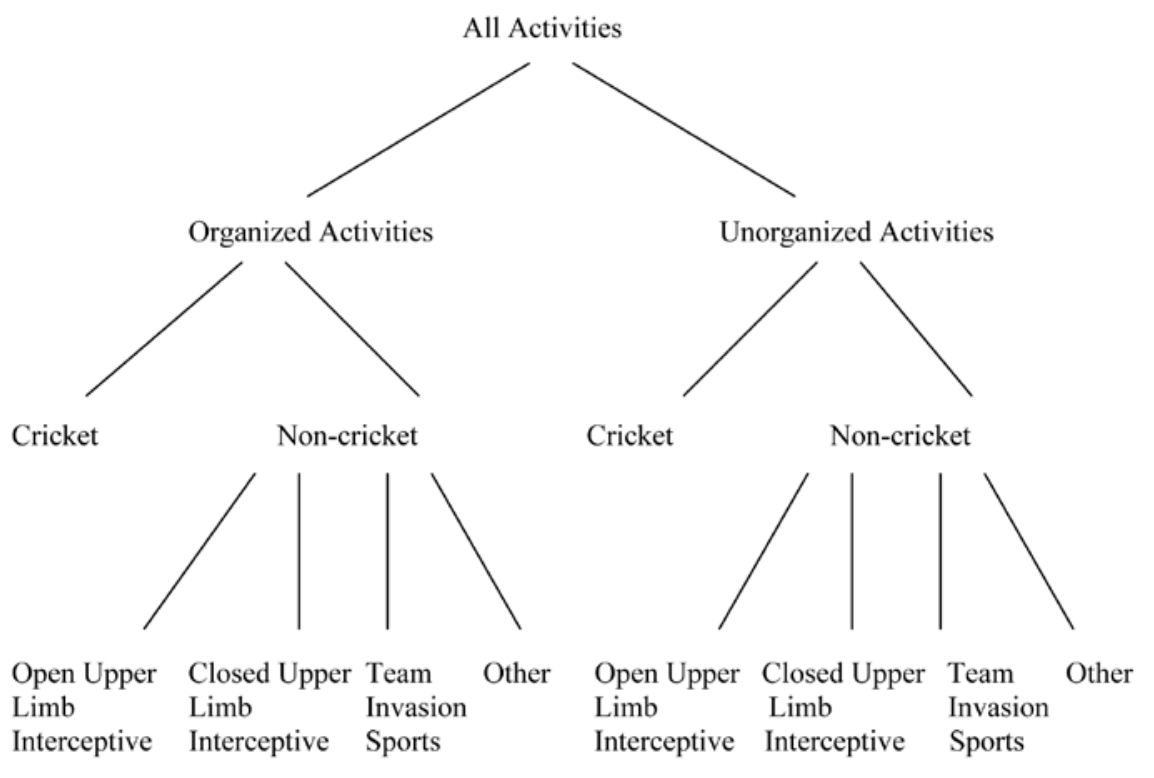

Figure 1 - Taxonomy used for the division of practice hours. 
were compared using a series of two-way (skill $\times$ age) ANOVAs with the sources of any significant effects being sought using the Tukey HSD procedure. Stepwise discriminant function analyses (with $F$ value set between .05 and .10 and the hours spent in different types of organized and unorganized sporting activity as input) were conducted to determine which of the practice measures provided the best discrimination between the different skill groups. Cross-validation was then performed using the "leave-one-out classification" procedure.

Predictors of Anticipatory Skill. Two multiple regression analyses were conducted to determine what practice variables were most predictive of anticipatory task performance. The first analysis used prediction accuracy for ball type achieved under the release condition temporal occlusion as the criterion variable and five predictor variables, namely, age and the hours spent separately in organized cricket, unorganized cricket, organized sports other than cricket, and unorganized sports other than cricket (as per the third tier of Figure 1). Using a stepwise approach, hours accumulated in organized contexts (cricket and noncricket) were entered first, followed by age (hierarchical), and thereafter the remaining unorganized practice variables were entered in a stepwise fashion. The variance in anticipatory performance that could be accounted for by practice variables was ascertained after first removing the variance that could be explained simply by participant age. The second analysis used the same predictor variables and approach but with accuracy in anticipating ball length (under the release temporal occlusion condition) as the criterion variable.

\section{Results}

\section{Anticipatory Skill}

Prediction of Ball Type. Figure 2 plots prediction accuracy for ball type (inswinger or outswinger) as a function of age for the skilled and less skilled players. Analysis of the participants' accuracy at the point of release $(\mathrm{R})$ revealed a significant effect for skill level, $F(1,96)=4.70, p<.05, \eta_{\mathrm{p}}^{2}=.05$, and age, $F(2$, 96) $=12.22, p<.01, \eta_{\mathrm{p}}{ }^{2}=.20$, but no interaction between these two factors, $F(2$, $96)=0.46, p>.05, \eta_{\mathrm{p}}{ }^{2}=.01$. The skilled players $(M=54.81, S D=22.71)$ were significantly more accurate than their lesser skilled counterparts $(M=45.75, S D=$ 21.22). The adults $(M=60.87, S D=19.69)$ and U20 players $(M=56.91, S D=$ 20.28) were superior to the U15 players $(M=38.41, S D=20.42)$, but did not differ from each other.

When prediction was compared between FFI and R, only the skilled adult players, $t(12)=2.57, p<.05$, and the skilled U20 players, $t(17)=1.86, p<.05$, were shown to be able to significantly increase prediction accuracy across this time period. For both these skilled groups, prediction levels at $\mathrm{R}$ were significantly above chance level $(p<.05)$, indicating a capability to pick up information in the time period immediately before ball release. For the U15 skilled players and the less skilled players of all ages, there was no comparable evidence of advance information pickup. These groups showed neither significant improvement from FFI to R nor prediction levels at R that were significantly greater than the $50 \%$ guessing levels. 


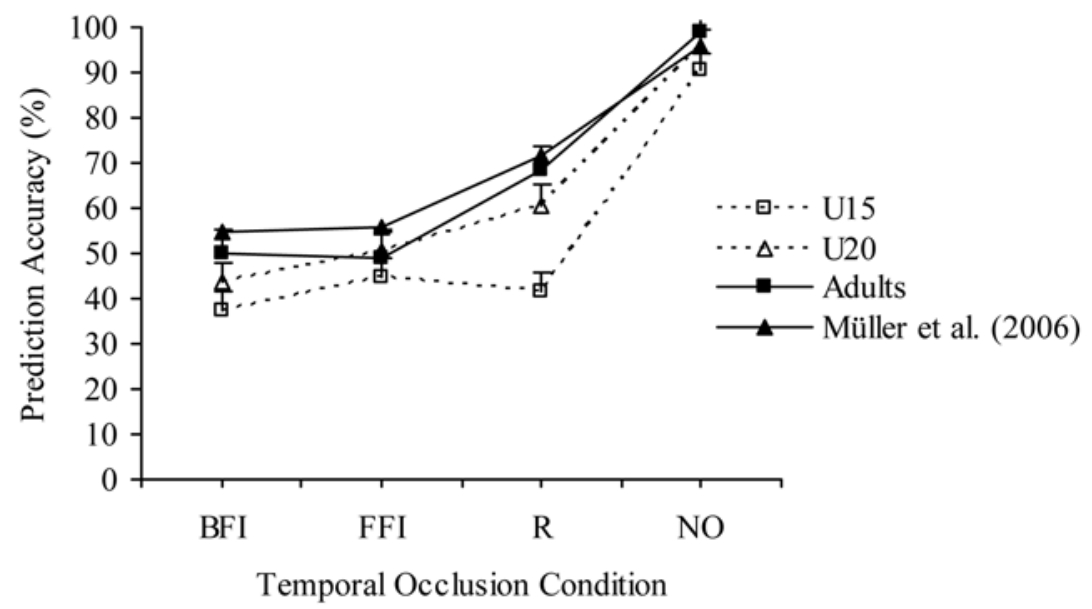

(a)

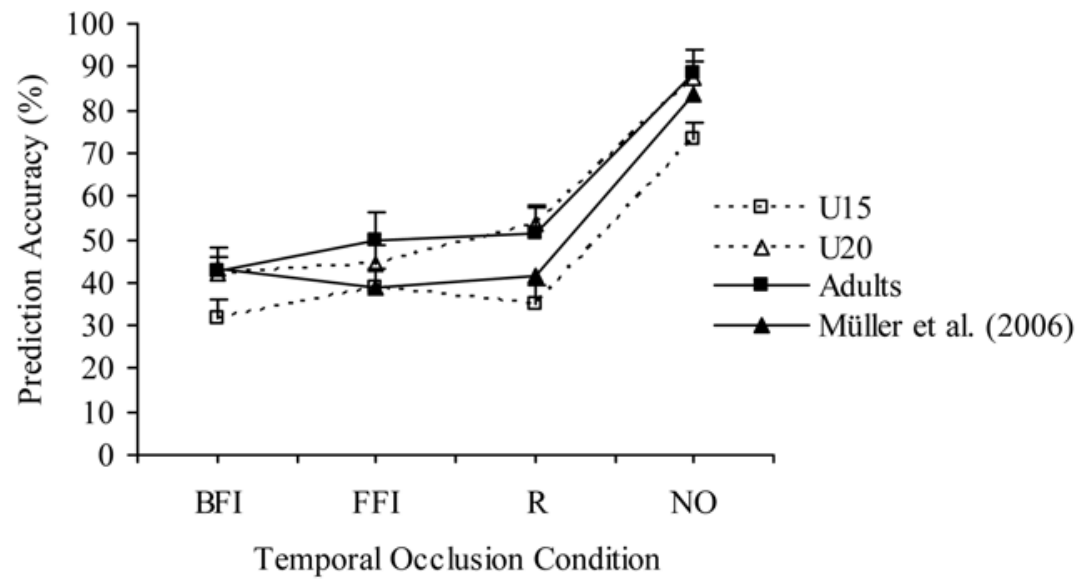

(b)

Figure 2 - Prediction accuracy of ball type as a function of age and time of occlusion for (a) skilled batsmen and (b) lesser skilled batsmen. Error bars represent standard error. The results of Müller et al. (2006) are used for comparative analysis. The skilled adult group prediction levels at both the $\mathrm{R}$ and $\mathrm{NO}$ conditions were significantly above the chance level of $50.0 \%(p<.05)$. The skilled U20 group's prediction levels exceeded chance levels under the $\mathrm{R}$ condition. For all other groups, prediction performance only significantly exceeded chance levels under the NO condition. 


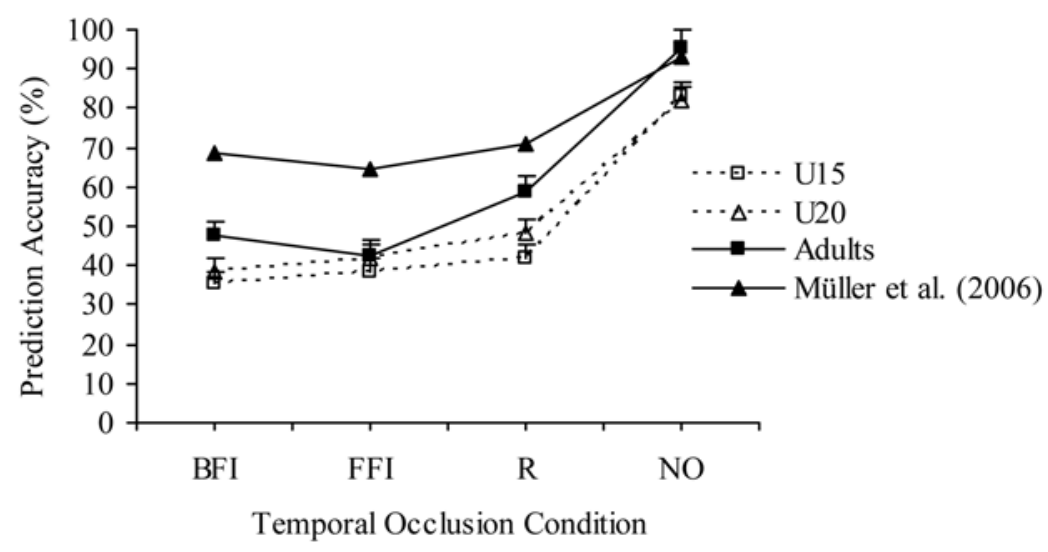

(a)

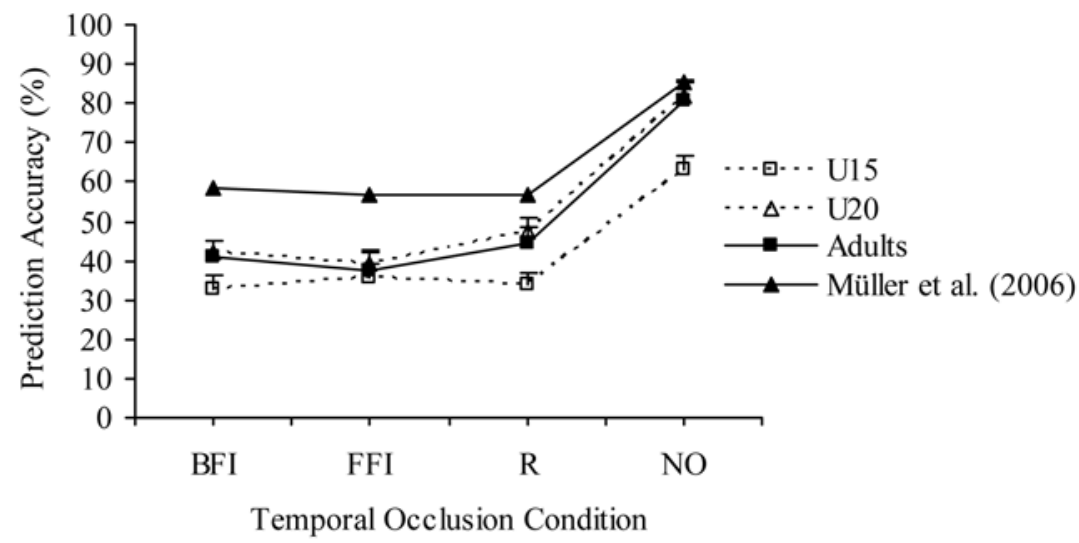

(b)

Figure 3 - Prediction accuracy of ball length as a function of age and time of occlusion for (a) skilled batsmen and (b) lesser skilled batsmen. Error bars represent standard errors. The results of Müller et al. (2006) are used for comparative analysis. For all participant groups, prediction accuracy only significantly exceeded guessing levels of $66.7 \%$ under the NO condition.

Prediction of Ball Length. There were significant main effects for skill level, $F(1,96)=6.44, p<.05, \eta_{\mathrm{p}}{ }^{2}=.06$, and age, $F(2,96)=8.12, p<.01, \eta_{\mathrm{p}}{ }^{2}=.15$, in the prediction of length at $\mathrm{R}$ but no interaction between these two factors, $F(2,96)$ $=1.64, p>.05, \eta_{\mathrm{p}}{ }^{2}=.03$ (see Figure 3 ). The skilled players $(M=48.40, S D=$ 16.83) were significantly better than their lesser skilled counterparts $(M=41.33$, $S D=14.48$ ). The adult and U20 groups (adult $M=52.54, S D=15.98 ; \mathrm{U} 20 M=$ 
$47.81, S D=14.84$ ) were superior to the U15 age group (U15 $M=38.00, S D=$ 14.68), but did not differ from each other.

In relation to evidence for information pickup in the FFI-R period only two groups - the skilled adults $[t(12)=3.12, p<.01]$ and the less-skilled U20 players $[t(19)=1.86, p<.05]$ - showed significant improvements in prediction accuracy from the FFI to R occlusion conditions; however neither group, nor any other group, achieved a prediction accuracy at $\mathrm{R}$ that significantly exceeded the guessing level of $66.7 \%$.

\section{Practice and Developmental Histories}

\section{Involvement in Organized Sporting Activity}

Total Number of Organized Sports. Analysis of the total number of organized sports each participant had experienced revealed no main effects for skill level, $F(1,96)=0.46, p>.05, \eta_{\mathrm{p}}{ }^{2}=.005$, or age, $F(2,96)=0.33, p>.05, \eta_{\mathrm{p}}{ }^{2}=.02$, and no interaction between these two factors, $F(2,96)=0.26, p>.05, \eta_{\mathrm{p}}{ }^{2}=.005$. The number of sports experienced during development did not differ between the skill groups at any of the U15, U20, and adult ages (see Table 1), suggesting that it is other factors that are critical for the development of batting expertise.

Hours Accumulated in Organized Cricket. There were significant main effects for skill level, $F(1,96)=30.44, p<.01, \eta_{\mathrm{p}}{ }^{2}=.24$, and age, $F(2,96)=25.10, p<$ $.01, \eta_{\mathrm{p}}{ }^{2}=.34$, as well as a significant skill level $\times$ age interaction, $F(2,96)=3.64$, $p<.05, \eta_{\mathrm{p}}^{2}=.07$ for hours spent playing organized cricket (see Figure 4a). Although the extent of the skill group differences was least pronounced among the U20 sample, and responsible for the significant interaction effect observed, across all age groups the differences in hours of commitment to organized cricket between the highly skilled and lesser skilled groups was marked. By as early as age U15, the skilled group's investment was approximately three times that of the lesser skilled group and by the adult age group the highly skilled group had accumulated more than 4000 more hours of experience in organized cricket than had the lessskilled performers (see Table 1).

Hours Accumulated in Organized Sports Other Than Cricket. There was no main effect for skill level, $F(1,96)=.001, p>.05, \eta_{\mathrm{p}}{ }^{2}=.00$, and no interaction between skill and age, $F(2,96)=.94, p>.05, \eta_{\mathrm{p}}^{2}=.02$, for hours of noncricket sport experience (see Figure $4 \mathrm{~b}$ ). This measure was affected significantly by age, however, $F(2,96)=8.54, p<.01, \eta_{\mathrm{p}}{ }^{2}=.15$, with the $\mathrm{U} 20$ and adult groups accumulating more hours of participation than the U15 group.

More-detailed examination of the types of organized sports, other than cricket, that were experienced by the participants revealed evidence of some types of activities for which there were skill-related differences in developmental experience and practice history. A main effect for skill level was found in relation to the number of hours accumulated in organized, closed upper limb interceptive sports, $F(1,96)=3.84, p=.05, \eta_{\mathrm{p}}{ }^{2}=.04$. The skilled group's investment was significantly greater than the lesser skilled group in this type of activity (skilled $M=$ 207.42, $S D=700.50$; lesser skilled $M=12.10, S D=48.71$ ), in large part due to a substantial commitment of some of the skilled batsmen to playing golf, but the 


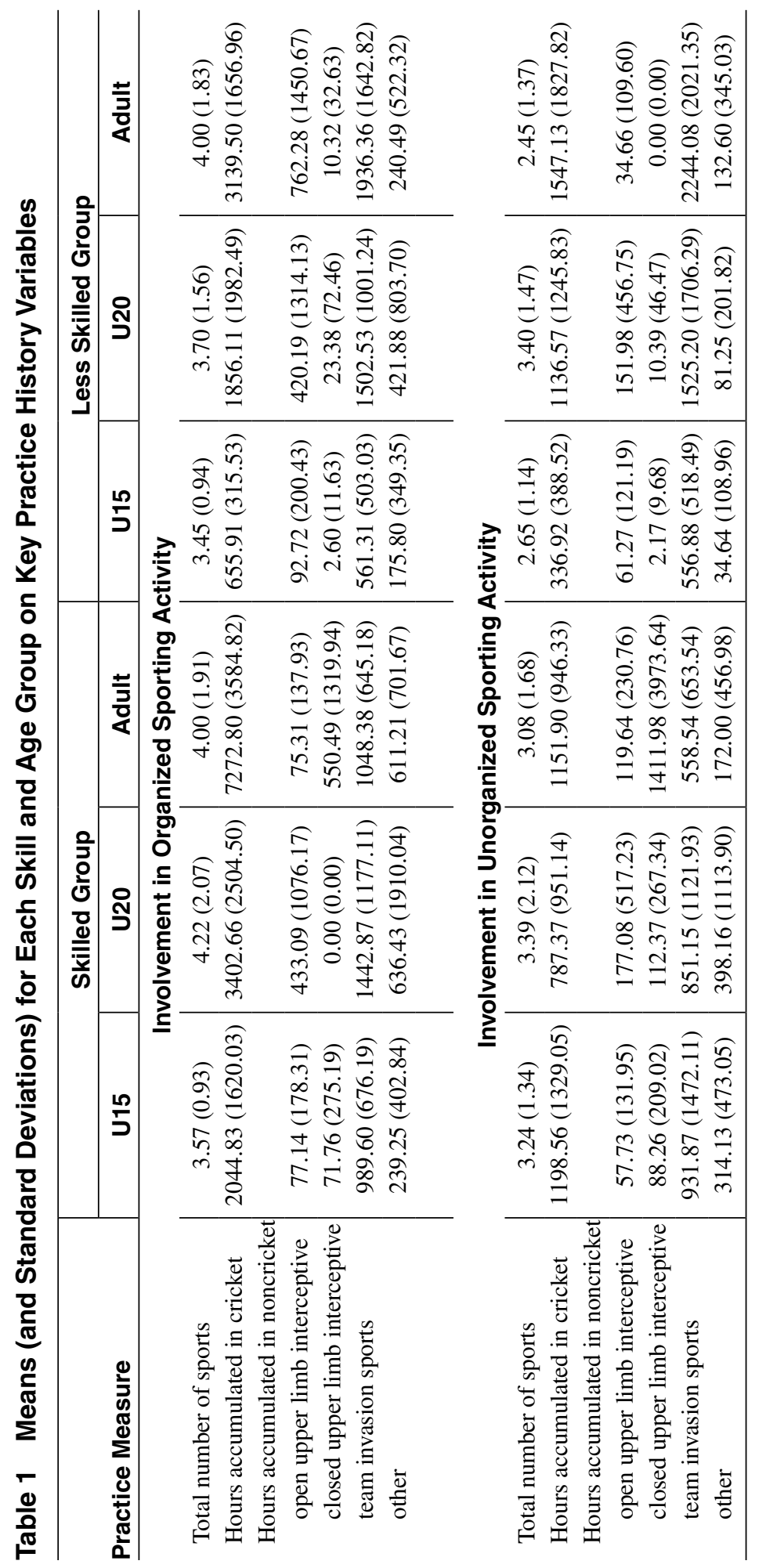




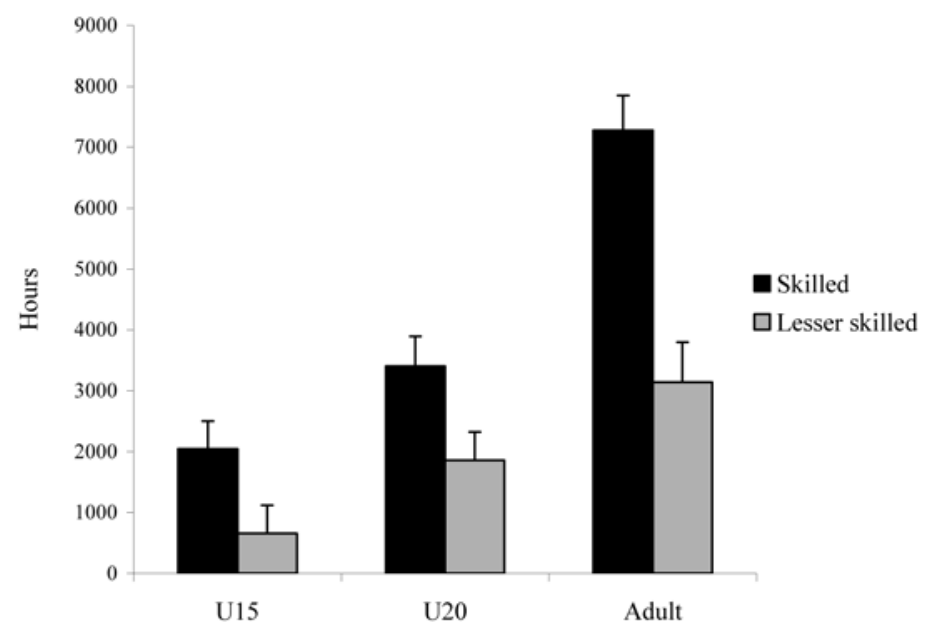

(a)

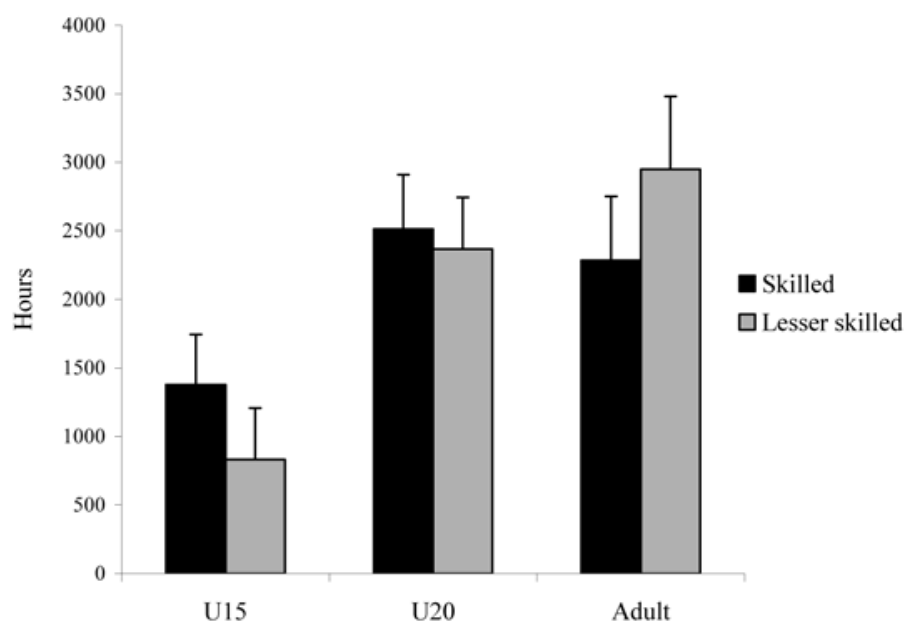

(b)

Figure 4 - Hours accumulated (a) in organized cricket and (b) in organized sports other than cricket for groups of skilled and lesser-skilled batsmen of U15, U20, and adult age. Bars represent standard errors. 
total hours accumulated were nevertheless small compared with those accumulated in cricket. A significant skill level $\times$ age interaction was also evident in relation to the hours accumulated in organized team invasion ball sports, $F(2,96)=$ $3.53, p<.05, \eta_{\mathrm{p}}{ }^{2}=.07$. The trend was for reduced involvement by the highly skilled cricket batsmen with age to the point of having less accumulated involvement in team sports by adulthood compared with the less skilled controls. The skilled U15 group had a greater investment than the lesser skilled U15 group in organized team invasion ball sports, the skilled and lesser skilled U20 groups had comparable investment and the adult skilled cricket group had accumulated significantly less hours in team ball sports than their less skilled cricket counterparts (see Table 1). Unsurprisingly, the skilled players reduced their involvement in other sports as they began to specialize in their primary sport. Skill main effects and skill $\times$ age interactions were not evident in relation to either the hours accumulated in organized open upper limb interceptive sport or the hours accumulated in other sports not requiring either interception or team-based invasion.

\section{Involvement in Unorganized Sporting Activity}

Total Number of Unorganized Sports. There were no significant main effects for skill level, $F(1,96)=0.89, p>.05, \eta_{\mathrm{p}}{ }^{2}=.01$, or age, $F(2,96)=1.42, p>.05$, $\eta_{\mathrm{p}}{ }^{2}=.03$, and there was no significant skill level $\times$ age interaction $F(2,96)=0.37$, $p>.05, \eta_{\mathrm{p}}{ }^{2}=.01$, in the total number of unorganized sports experienced by the different participants. The number of unorganized sports experienced did not differ between the highly skilled and less skilled cricket batsmen at the U15, U20, or adult ages (Table 1).

Hours Accumulated in Unorganized Cricket. There were no significant main effects for skill level, $F(1,96)=0.03, p>.05, \eta_{\mathrm{p}}{ }^{2}=.00$, or age, $F(2,96)=1.91, p$ $>.05, \eta_{\mathrm{p}}{ }^{2}=.04$, in relation to the hours participants had spent playing unorganized cricket; however, there was a significant skill level $\times$ age interaction, $F(2,96)=$ $3.57, p<.05, \eta_{\mathrm{p}}{ }^{2}=.07$. As Figure 5a reveals, the skilled batsmen in the U15 age grouping had invested approximately three times as many hours in unorganized forms of cricket than had the lesser skilled batsmen, but by the U20 age the situation was reversed, with the lesser skilled batsmen's investment being greater than that of the skilled batsmen. This trend for greater investment by the less skilled also continued in the comparisons drawn at the adult age (see Table 1). The apparent decrease in accumulated hours spent in unorganized cricket from U15 to U20 to adult for the skilled group is a consequence of each representing a different cohort of participants and highlights some of the limitations inherent in the use of cross-sectional rather than longitudinal research designs.

Hours Accumulated in Unorganized Sports Other Than Cricket. There were no main, $F(1,96)=0.08, p>.05, \eta_{\mathrm{p}}{ }^{2}=.001$, or interactive effects for skill, $F(2$, 96) $=0.65, p>.05, \eta_{\mathrm{p}}{ }^{2}=.01$, on the hours accumulated in unorganized sports other than cricket. A main effect was apparent for age, $F(2,96)=4.00, p=.05, \eta_{p}{ }^{2}$ $=.06$, owing to the accumulation of more participation hours by the groups of adult age than those who were U15 (see Table 1 and Figure 5b).

In terms of the various categories of unorganized sports other than cricket, no significant skill main or interactive effects were observed in relation to the hours 


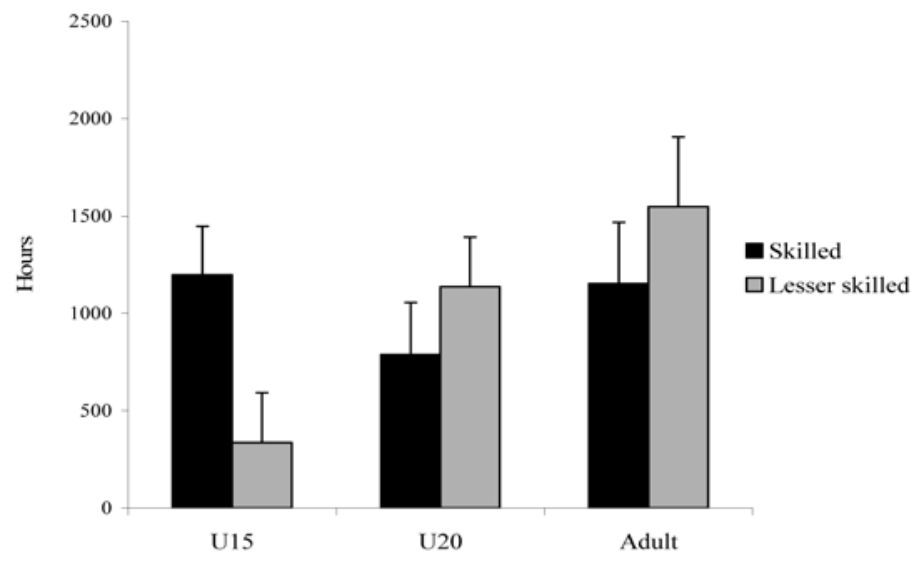

(a)

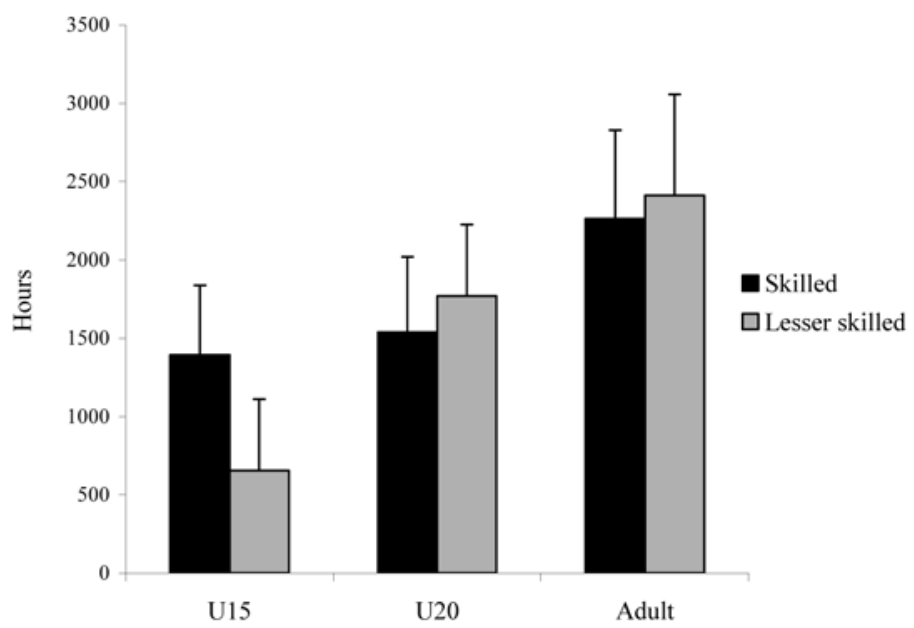

(b)

Figure 5 - Hours accumulated (a) in unorganized cricket and (b) in unorganized sports other than cricket for groups of skilled and lesser-skilled batsmen of U15, U20, and adult age. Bars represent standard errors. 
accumulated in open upper limb interceptive sports, in closed upper limb interceptive sports, and in other sports without interceptive or team invasion characteristics. A significant skill level $\times$ age interaction was found, however, in relation to the hours accumulated in unorganized team invasion ball sports, $F(2,96)=4.61$, $p<.05, \eta_{\mathrm{p}}{ }^{2}=.09$. In keeping with the observation in relation to organized activities of this type, the skilled batsmen in the U15 age grouping had an average investment that was significantly greater than their lesser skilled counterparts, but this trend was reversed within the U20 and the adult groups (see Table 1).

\section{Practice Variables Discriminating Skill Group}

A discriminant function analysis was used to determine which variables most strongly discriminated between the skill levels and to determine how accurately membership of these different groups could be predicted. A significant discriminant function for prediction of skill group membership was derived, $\chi^{2}(1)=16.40$, $p<.01$, with accumulated hours in organized cricket found to be the sole contributor to the significant function $(F=17.92 ; d f 1,100 ; p<.001$; group centroids: skilled $=0.41$; less skilled $=-0.43$ ). The model accurately predicted the group membership of $64.7 \%$ of cases $-50.0 \%$ of the skilled batsmen and $80.0 \%$ of the lesser skilled batsmen were categorized successfully. Cross-validation revealed that successful classification of skill level could be maintained $(63.7 \%)$, with $48.1 \%$ of the skilled batsmen and $80.0 \%$ of the lesser skilled batsmen still categorized correctly.

\section{The Relationship Between Anticipatory Skill and Practice History}

\section{Prediction of Anticipation of Ball Type}

The multiple regression analysis in which accuracy in predicting ball type from the anticipation task when the display was occluded at the point of release was the criterion variable, and age plus the hours accumulated in both organized and unorganized cricket and organized and unorganized sports other than cricket were the predictor variables, produced significant equations from the first two stages of the analysis. In the first (stepwise) stage of the analysis, hours accumulated in organized cricket was found to account for $13.3 \%$ of the variance in ball type anticipation, $R=.36 ; R^{2}=.13$; adjusted $R^{2}=0.12 ; F(1,100)=15.37, p<.001$. In the second (hierarchical) stage of the analysis, inclusion of age increased explained variance by a further $6.9 \%, R=.45 ; R^{2}=.20$; adjusted $R^{2}=0.19 ; F(1,99)=8.52$, $p<.005$. When the remaining practice variables were entered in the third stage of the analysis (stepwise), all failed to significantly improve prediction of the anticipation scores.

\section{Prediction of Anticipation of Ball Length}

Hours accumulated in organized cricket explained $11.4 \%$ of the variance in ball length anticipation, $R=.34 ; R^{2}=.11$; adjusted $R^{2}=0.11 ; F(1,100)=12.92, p<$ .005 , whereas the addition of age increased explained variance by a further $4.7 \%$, 
$R=.40 ; R^{2}=.16$; adjusted $R^{2}=0.14 ; F(1,99)=5.54, p<.001$. None of the other practice variables permitted further improvements in the prediction of the ball length anticipation scores.

\section{Discussion}

We examined the relationship between anticipatory skill in cricket batting and the developmental practice histories of groups of skilled and lesser skilled batsmen of three different ages and stages of sport development. The study proceeded in three parts. First, the ability of participants at different age and skill levels to anticipate the type and length of delivery being bowled by a medium-fast bowler was assessed using an established laboratory-based temporal occlusion task (from Müller et al., 2006). Second, the total hours of developmental sporting investment were detailed across the different skill and age groups using retrospective recall to determine what developmental/practice variables differentiated the various cohorts. Finally, an attempt was made to converge the anticipatory and practice history data to find those aspects of the participant's practice histories that were most predictive of their anticipatory skills.

The anticipation task revealed some important insights into the evolution of the capability to use advance sources of information to predict both future ball type and length. Systematic skill and age group differences were revealed on both the type and length predictions, with the skilled players outperforming the lessskilled players and the adult and U20 players outperforming the U15 players. In terms of the time course of advance information pickup, skilled adult and U20 players showed a capability to use information in the period from FFI to R to improve prediction accuracy of ball type, and to a level better than guessing. This capability was not evident in either the younger (U15) skilled group or in the less skilled groups of any age. Adult skilled batsmen, plus the less skilled U20 players, also showed a capability to significantly improve their accuracy in predicting ball length from information available in the period from the bowler's front-foot impact through to release (see Figures $2 \mathrm{a}$ and $3 \mathrm{a}$ ), but not to a level that permitted prediction accuracy to significantly exceed the stringent guessing level set for length judgments of $66.7 \%$. The observation that highly skilled but not less skilled adult aged players can anticipate ball type through information pickup in the FFI$\mathrm{R}$ period is consistent with the findings of Müller et al. (2006). The failure to show prediction accuracy levels for ball length judgments significantly better than guessing levels may be primarily a consequence of adopting a stringent criterion for determining chance levels based on the assumption of the participants knowing the relative frequency of full and short deliveries and adopting a response bias in favor of the more prevalent full-length deliveries (cf. Müller et al., 2006). If a less stringent criterion is adopted (such as the 50\% level used for type judgments), then a conclusion could also be reached of a capability of highly skilled batsmen to also pick up advance information regarding ball length.

The presence of well-developed anticipatory skills for ball type in skilled adult and U20 players but not in skilled U15 players is of interest, and there are a number of possible reasons as to why well-developed anticipatory skills were not evident in the highly skilled younger batsmen. One possibility is that anticipatory 
skills may be less important in junior competition levels as the bowling speeds are generally slower and may not impose sufficient time constraints to make advance judgments on the basis of prerelease information necessary for success. In the junior ranks, reliance on early ball flight information may be sufficient to allow interceptive movements to be planned and executed in time and other factors such as physical size and strength may be more important contributors to batting success than anticipatory ability. A second possibility is that, while anticipation may be potentially important for batting even in the junior age groupings, the ability of players to detect subtle postural information from the bowler may require the accumulation of repeated perceptual exposure to bowlers' movement patterns of such volume that there is simply insufficient time to accrue the request experience by age 15-20 years. In this regard, the exposure to large amounts of cricket-specific practice in unorganized settings that skilled players experience during their early years may be ultimately valuable in supplementing the perceptual skill learning that occurs within organized cricket settings. The acquisition of the skill of recognizing subtle postural information for anticipation may have a much longer developmental time frame than the acquisition of pattern recognition skills, such as those used in team games, that require understanding of more global relationships between players (Ward \& Williams, 2003).

Some tentative evidence in support of the development of expert anticipation skills requiring the accumulation of many years of experience can be derived from inspection of the comparative performance of the different age groupings for the skilled and the lesser skilled batsmen. While the highly skilled U15 cricket batsmen were not able to provide compelling evidence of an ability to effectively use advance information, it is nevertheless noteworthy that, for both the prediction of ball type and length, accuracy levels at the release (R) occlusion condition showed a systematic relationship to age for the skilled participants, with the mean accuracy of the skilled adult group being greater than that of the U20 skilled group, which was, in turn, greater than that of the U15 skilled group. Consistent with the observations on anticipation in badminton (Abernethy, 1988), no comparable age progressions were evident for the less skilled groups, indicating that the improvements evident for the skilled group are most likely experience related and not simply due to biological maturation. To examine this proposition further, however, requires consideration of the data on the practice histories of the players and the relationship between practice experiences and anticipatory performance.

The accumulated hours spent in organized cricket emerged from the second part of the study as the main discriminator of both the skill groups and the age groups. Across all three age groups examined, the highly skilled players selfreported having accumulated 2-3 times more hours of organized cricket experience than their age-matched controls. The total number of hours accumulated in unorganized cricket activity was also greater for the highly skilled players in the U15 age group, but not among the older age groups. Likewise, the number of hours of experience accumulated in invasion sports (both organized and unorganized) was greater for the skilled cricket batsmen at the U15 age but less than that for the less skilled players for the U20 and adult ages, consistent with the transition of the older skilled players into the investment stage of sports participation (Côté, 1999; Côté \& Hay, 2002). No evidence was apparent in this study for highly skilled players experiencing a greater range of sports (either 
organized or unorganized) and similarly no evidence was revealed to suggest greater exposure by highly skilled batsmen to other upper limb interceptive activities like baseball or tennis that might have conceivably offered the opportunity for perceptual, motor, or strategic transfer (cf. Schmidt \& Wrisberg, 2000).

The observations that highly skilled batsmen (of adult and U20 age) have anticipatory skills superior to those of less skilled players and that highly skilled batsmen (of all ages) have accrued significantly greater amounts of cricket-specific experience than their less skilled counterparts suggest that anticipatory skill and the volume of sport-specific experience might be closely associated. In most texts on the topic of skill learning for sports like cricket, the accumulation of large amounts of task-specific experience is seen as being essential for providing the requisite conditions to permit the learning of anticipatory skill to occur. For this reason, we expected that individual differences in anticipatory ability (as measured using the temporal occlusion task of Müller et al., 2006) would be able to be predicted with considerable accuracy from one or a combination of developmental practice variables. The findings, from the third part of this study using a multiple regression approach, were consequently unexpected. The regression analyses indicated that although hours accumulated in organized cricket could significantly predict anticipatory performance, the variance in anticipatory performance explained by accrued practice hours, independent of the variance simply explicable by age, was a relatively modest $11-13 \%$.

There are a number of possible explanations as to why the accrued practice hours provided only a weak prediction of anticipatory skill. First, our measure of anticipatory skill was an incomplete one and it may be that this artificially limited the capacity to demonstrate a stronger link between anticipation and practice. While the anticipation measure used in this study provided an indication of individual differences in the ability to pick up information from the bowler's prerelease movement kinematics - and this is an ability known to be reliably linked to skilled performance-we did not assess each participant's ability to use contextual information/subjective probabilities for anticipation, nor did we assess capability to extract predictive information from early ball flight. Both these components of effective anticipation are known to differ between players of different skill levels (Abernethy, Gill, Parks, \& Packer, 2001; Müller \& Abernethy, 2006). Given there is a likely progression, as the bowling event unfolds, for judgments to be made on the basis of probability information as the bowler commences his runup, on the basis of prerelease kinematic information as the bowler prepares to deliver the ball, and then on ball flight information as soon as the ball is released, future studies measuring anticipatory/perceptual skills in cricket may need to also measure participant sensitivity to probability information and early ball flight information in addition to sensitivity to prerelease kinematics. Our anticipation test also used only a single bowler (of adult age and high skill) and there would be clear benefits to increasing both the number and the age and skill ranges of the bowlers to improve the representativeness of the sampling of anticipatory ability that is possible.

Second, it may be that the measures of practice experience that we have taken, while not atypical of those used in studies of the developmental histories of experts, may be insufficiently sensitive or fine grained to capture critical elements 
of the practice experience that contribute to the acquisition of anticipatory skill. It may be that anticipatory skill is more related to the type of cricket-specific practice that individuals experience rather than to the amount of hours of experience, and this argues for increased use of approaches that more precisely measure the microstructure of practice (cf. Deakin \& Cobley, 2003). In future, researchers should seek to further differentiate types of cricket-specific practice (e.g., solitary batting practice, net-based practice with a bowler, net-based practice with a ball machine, game-context practice, video-based practice) and make clear, in organized settings, a distinction between the hours of experience accumulated in and outside of actual matches.

\section{Acknowledgments}

This investigation was supported by a research grant provided by the Cricket Australia Sports Science/Medicine Advisory Group. The authors are grateful for the significant contribution made by the participants, research assistants, Jason Berry, the Australian Institute of Sport Skill Acquisition staff, the Australian Institute of Sport Cricket Program, and the ACT and Queensland Cricket Associations.

\section{References}

Abernethy, B. (1988). The effects of age and expertise upon perceptual skill development in a racquet sport. Research Quarterly for Exercise and Sport, 59(3), 210-221.

Abernethy, B., Gill, D.P., Parks, S.L., \& Packer, S.T. (2001). Expertise and perception of kinematic information and situational probability information. Perception, 30, 233252.

Abernethy, B., \& Russell, D.G. (1984). Advance cue utilisation by skilled cricket batsmen. Australian Journal of Science and Medicine in Sport, 16(2), 2-10.

Baker, J., Coté, J., \& Abernethy, B. (2003). Sport specific training, deliberate practice and the development of expertise in team ball sports. Journal of Applied Sport Psychology, $15,12-25$.

Bartlett, F.C. (1947). The measurement of human skill. British Medical Journal, June 14, $835-838$.

Cohen, J. (1988). Statistical power analysis for the behavioral sciences (2nd ed.). Hillsdale, NJ: Laurence Erlbaum.

Côté, J. (1999). The influence of the family in the development of talent in sport. The Sport Psychologist, 13, 395-417.

Côté, J., Baker, J., \& Abernethy, B. (2007). Practice and play in the development of sport expertise. In G. Tenenbaum \& R.C. Eklund (Eds.), Handbook of sport psychology (3rd ed., pp. 184-202). New York: Wiley.

Côté, J., \& Hay, J. (2002). Children's involvement in sport: A developmental perspective. In J.M. Silva \& D. Stevens (Eds.), Psychological foundations of sport (pp. 484-502). Boston: Allyn and Bacon.

Côté, J., Ericsson, K.A., \& Law, M.P. (2005). Tracing the development of athletes using retrospective interview methods: A proposed interview and validation procedure for reported information. Journal of Applied Sport Psychology, 17, 1-19.

Deakin, J.M., \& Cobley, S. (2003). A search for deliberate practice: An examination of the practice environments in figure skating and volleyball. In I.J. Starkes \& K.A. Ericsson (Eds.), Expert performance in sports: Advances in research on sport expertise (pp. 115-132). Champaign, IL: Human Kinetics. 
Ericsson, K.A. (2003). Development of elite performance and deliberate practice. In J. Starkes \& K.A. Ericsson (Eds.), Expert performance in sports: Advances in research on sport expertise (pp. 49-81). Champaign, IL: Human Kinetics.

Ericsson, K.A., Krampe, R.T., \& Tesch-Romer, C. (1993). The role of deliberate practice in the acquisition of expert performance. Psychological Review, 100(3), 363-406.

Helsen, W.F., Hodges, N.J., Van Winckel, J., \& Starkes, J.L. (2000). The roles of talent, physical precocity and practice in the development of soccer expertise. Journal of Sports Sciences, 18, 727-736.

Helsen, W.F., Starkes, J.L., \& Hodges, N.J. (1998). Team sports and the theory of deliberate practice. Journal of Sport \& Exercise Psychology, 20, 12-34.

Hodges, N.J., \& Starkes, J.L. (1996). Wrestling with the nature of expertise: A sport specific test of Ericsson, Krampe and Tesch-Romer's (1993) theory of 'deliberate practice'. International Journal of Sport Psychology, 25, 1-25.

Land, M.F., \& McLeod, P. (2000). From eye movements to actions: how batsmen hit the ball. Nature Neuroscience, 3, 1340-1345.

McLeod, P. (1987). Visual reaction time and high-speed ball games. Perception, 16, 4959.

Müller, S., \& Abernethy, B. (2006). Batting with occluded vision: An in situ examination of the information pick-up and interceptive skills of high- and low-skilled cricket batsmen. Journal of Science and Medicine in Sport, 9, 446-458.

Müller, S., Abernethy, B., \& Farrow, D. (2006). How do world-class cricket batsmen anticipate a bowler's intention? Quarterly Journal of Experimental Psychology, 59, 2162-2186.

Musch, J., \& Grondin, S. (2001). Unequal competition as an impediment to personal development: A review of the relative age effect in sport. Developmental Review, 21, 147-167.

Schmidt, R.A., \& Wrisberg, C.A. (2000). Motor learning and performance: A problembased learning approach. Champaign, IL: Human Kinetics.

Starkes, J.L., \& Ericsson, K.A. (2003). Expert performance in sports: Advances in research on sport and expertise. Champaign: IL, Human Kinetics.

Tenenbaum, G., Sar-El, T., \& Bar-Eli, M. (2000). Anticipation of ball location in low and high-skill performers: A developmental perspective. Psychology of Sport and Exercise, 1, 117-128.

Renshaw, I., \& Fairweather, M.M. (2000). Cricket bowling deliveries and the discrimination ability of professional and amateur batters. Journal of Sports Sciences, 18, 951-957.

Ward, P., \& Williams, A.M. (2003). Perceptual and cognitive skill development in soccer: The multidimensional nature of expert performance. Journal of Sport \& Exercise Psychology, 25, 93-111.

Williams, A.M., \& Ericsson, K.A. (2005). Perceptual-cognitive expertise in sport: Some considerations when applying the expert performance approach. Human Movement Science, 24, 283-307.

Manuscript submitted: September 21, 2007

Revision accepted: August 10, 2008 